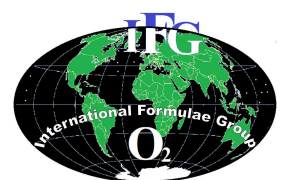

Available online at http://www.ifgdg.org

Int. J. Biol. Chem. Sci. 10(3): 993-1006, June 2016

International Journal

of Biological and

Chemical Sciences

ISSN 1997-342X (Online), ISSN 1991-8631 (Print)

Original Paper http://ajol.info/index.php/ijbcs http://indexmedicus.afro.who.int

\title{
Effets des associations culturales sur la dynamique de population des principaux insectes ravageurs et la production en graines de Jatropha curcas L. au Sud-Bénin
}

\author{
Alexis ONZO $^{1 *}$, Moustapha Sabi SEIDOU ${ }^{1}$, Nouhoun ZOUMAROU-WALLIS ${ }^{1}$, \\ Benjamin Dossa DATINON ${ }^{2}$ et Manuele TAMÓ ${ }^{2}$ \\ ${ }^{1}$ Faculté d'Agronomie, Département des Sciences et Techniques de Production Végétale, \\ Université de Parakou, B.P. 123, Parakou, Bénin. \\ ${ }^{2}$ Biological Control Centre for Africa, International Institute of Tropical Agriculture, \\ 08 B.P. 0932, Cotonou, Benin. \\ *Auteur correspondant ; E-mail: onzalex@yahoo.com
}

\section{REMERCIEMENTS}

Les auteurs remercient l'Institut International d'Agriculture Tropicale (station du Bénin) pour son soutien financier et matériel à la réalisation de ce travail.

\section{RÉSUMÉ}

Jatropha curcas est l'une des cultures proposées actuellement pour la production de biocarburant, ce qui pourrait entraver les cultures vivrières. Il a alors été suggéré d'associer jatropha avec les cultures vivrières. L'effet des associations Jatropha+Maïs (JM), Jatropha+Niébé (JN), Jatropha+Manioc (JMc) et Jatropha seul (Js) a été évalué sur les populations des ravageurs de J. curcas en champ à Ouinhi au Sud-Est du Bénin. Les sept espèces d'insectes ravageurs recensées sont : Denticera divisella, Aulocophora africana, Calidea dregei, Lagria vilosa, Apion sp., Stomphastis thraustica et Clavigralla tomentosicollis dont les plus importantes sont : Apion sp., A. africana et $D$. divisella. Le niveau de population des ravageurs a varié suivant l'association et le stade phénologique de jatropha. Les pourcentages de feuilles attaquées sont de 56,0 $\pm 3 \% ; 52,0 \pm 3 \% ; 47,0 \pm$ $3 \%$ et $44,0 \pm 3 \%$, respectivement pour JM, JN, JMc et Js. Les rendements en graines de jatropha sont de 9,77 \pm $1,57 \mathrm{~g} ; 9,40 \pm 1,71 \mathrm{~g} ; 4,63 \pm 1,34 \mathrm{~g} ; 2,25 \pm 1,10 \mathrm{~g}$ par plant, respectivement pour Js, JM, JN et JMc. En général, l'association culturale a affecté négativement la productivité du jatropha notamment dans les associations Jatropha+Manioc et Jatropha+Niébé qu'il importe de déconseiller.

(c) 2016 International Formulae Group. All rights reserved.

Mots clés: Biocarburant, pourghère, associations culturales, insectes ravageurs de jatropha, Denticera divisella, rendement de jatropha.

\section{Effects of mixed cropping on the population dynamics of main insect pests and seed production by Jatropha curcas L. in Southern Benin}




\section{ABSTRACT}

Jatropha curcas is one of the crops currently proposed for the production of biofuel; however, this could hamper food crop production. Therefore, it was suggested to mixed-crop jatropha with food crops. In a field trial at Ouinhi, southeast-Benin, we evaluated the effects of the mixed-cropping of Jatropha + Maize (JM), Jatropha + Cowpea (JC), Jatropha + Cassava (JCa) and Jatropha alone (Ja) on populations of insect pests infesting $J$. curcas. The seven insect pest species recorded on jatropha were: Denticera divisella, Aulocophora Africana, Calidea dregei, Lagria vilosa, Apion sp., Stomphastis thraustica and Clavigralla tomentosicollis, among which the most important species were Apion sp., A. africana and D. divisella. Pest population levels varied with the crop association and the phenological stage of jatropha. Percentages of damaged leaves were $56.0 \pm 3 \% ; 52.0 \pm 3 \% ; 47.0 \pm 3 \%$ and $44.0 \pm 3 \%$, respectively for JM, JC, JCa and Ja. Jatropha seed yield per plant averaged $9.77 \pm 1.57 \mathrm{~g} ; 9.40 \pm 1.71 \mathrm{~g} ; 4.63 \pm 1.34 \mathrm{~g} ; 2.25 \pm 1.10 \mathrm{~g}$, respectively for Ja, JM, JC and JCa. In general, mix-cropping negatively affected jatropha productivity, especially when mixed with cowpea or cassava. Therefore, such crop associations should not be advised.

(C) 2016 International Formulae Group. All rights reserved.

Keywords: Biofuel, Jatropha curcas, mixed cropping, Denticera divisella, Insect pests of jatropha, jatropha yield.

\section{INTRODUCTION}

L'énergie constitue, de nos jours, une ressource fondamentale dans le développement d'un pays. C'est un secteur essentiel dans la vie socio-économique de toute nation. Cependant, de nombreux pays à travers le monde connaissent encore des problèmes d'insuffisance énergétique et dépendent, de ce fait, de l'extérieur pour leur approvisionnement en énergie électrique, en carburants d'origine pétrolière et en gaz domestique (Vignon, 2008). Afin de limiter cette dépendance vis-à-vis de l'extérieur et réduire la pollution atmosphérique liée à l'effet de serre induit par les combustibles d'origine fossile, plusieurs pays se sont intéressés au développement de sources d'énergie renouvelable, moins polluantes et moins coûteuses (Gandonou, 2007). Parmi ces sources d'énergie renouvelable, les bioénergies et en particulier les 'biocarburants' appelés encore 'agrocarburants' sont celles qui retiennent le plus l'attention de plusieurs gouvernements (Vignon, 2008). Au nombre des plantes utilisées pour la production du biodiesel, le pourghère, Jatropha curcas L. occupe une place de choix dans les pays tropicaux.

Jatropha curcas est un arbuste originaire de l'Amérique Centrale ou du Mexique (Aponte, 1978; Wilbur, 1997). C'est une plante dicotylédone appartenant à la famille des Euphorbiacées. Le jatropha se présente sous la forme d'un arbre ou arbuste de $2 \mathrm{~m}$ à plus de $10 \mathrm{~m}$ de hauteur. Sa multiplication peut être réalisée par graine ou par bouture. Souples quand elles sont jeunes, ses branches deviennent cassantes avec l'âge ; ce qui peut gêner les opérations de récolte. L'arbre donne des fruits de couleur jaune qui deviennent marron foncé en séchant. Les fruits renferment 1 à 3 graines riches en une l'huile visqueuse qui peut être utilisée dans la fabrication de bougies, de savons, de produits cosmétiques mais surtout comme un substitut au diesel (gas-oil). Cette propriété a des implications importantes dans la satisfaction de la demande en ressources énergétiques des ménages et surtout dans la quête mondiale de remplaçants renouvelables aux combustibles fossiles; la finalité de ceci étant la réduction de l'accumulation des gaz à effet de serre dans l'atmosphère en vue de la protection de la couche d'ozone (Kumar et Sharma, 2008).

Cependant, l'utilisation de jatropha pour la production du biocarburant apparaît comme un handicap majeur à la satisfaction de l'autosuffisance alimentaire, en particulier dans les régions d'Afrique sub-Saharienne. En effet, les espaces consacrés à la culture du jatropha constituent un manque à gagner pour l'agriculture traditionnelle, ce qui pourrait 
freiner son adoption (Dorin et Gitz, 2007). Pour contourner cette difficulté, les services de vulgarisation agricole du Bénin conseillent une association entre jatropha et les cultures vivrières.

Les expériences de cultures intercalaires de jatropha ne sont pas nombreuses mais ne semblent pas présenter de contre-indication particulière (Brittaine et Lutaladio, 2010), si ce n'est d'éviter son association avec une autre euphorbiacée telle que le manioc ou l'hévéa, le jatropha pouvant être une plante-hôte pour les mêmes bioagresseurs (Hountongninou, 2009). En effet, à côté des contraintes techniques et stratégiques, la culture du jatropha n'est pas exempte de contraintes phytosanitaires (Datinon et al., 2013). Bien qu'étant généralement reconnu comme une plante très résistante, le pourghère est sensible aux adventices, surtout lorsqu'il est encore au stade juvénile, les plants issus du semis direct étant plus affectés que ceux issus de boutures (Münch, 1986). Aussi, le pourghère n'est pas à l'abri des maladies. En effet, les champignons des genres Phytophtora, Phytium et Fusarium provoquent la pourriture des racines (Domergue et Pirot, 2008). De plus, malgré le fait que $J$. curcas possède des propriétés toxiques (Brittaine et Lutaladio, 2010), il n'est pas à l'abri des insectes ravageurs dont la pression augmente en monoculture (Otieno et Mwangi, 2005; Brittaine et Lutaladio, 2010). Ainsi, plusieurs espèces de l'ordre des Hétéroptères ont été identifiées au Nicaragua, piquant le fruit en y injectant un liquide qui dissout la graine (Legendre, 2008). En Inde, deux principales espèces causent de sérieux problèmes aux plants de J. curcas. Il s'agit de Pempelia morosalis Saalm Üller (Lepidoptera: Pyralidae) qui attaque les inflorescences, tisse des soies, creuse des galeries au niveau des tiges tendres et dévore les jeunes capsules; et de Scutellera nobilis (Heteroptera: Scutelleridae) qui provoque la chute des fleurs, l'avortement des fruits et la malformation des graines. Au Kénya, les espèces suivantes ont été identifiées: Calidea dregei Germar (Heteroptera: Scutelleridae), Planococcus sp. (Homoptera: Pseudococcidae), Aphtona sp (Coleoptera: Chrysomelidae), Stomphastis rupsen (Lepidoptera: Gracillariidae) (Otieno et Mwangi, 2009). D'autres insectes comme Leptoglossus sp. (Heteroptera: Coreidae), Achea janata Linnaeus (Lepidoptera: Noctuidae), Indarbela quadrinotata Walker et Oxycetonia versicolor Fabricius (Coleoptera: Cetoniidae) ont également été identifiés comme étant des ravageurs de J. curcas (Brittaine et Lutaladio, 2010). Au Bénin, de récentes études portant sur l'entomofaune associée à la culture de $J$. curcas a montré que Denticera divisella Duponchel (Lepidoptera : Pyralidae) est le micro-lépidoptère le plus dévastateur des fleurs et fruits de Jatropha au Sud- Bénin (Togbossy, 2012 ; Adebiyi, 2013).

Etant reconnu que la polyculture héberge un grand nombre d'espèces d'insectes comprenant aussi bien des prédateurs, des parasitoïdes que des ravageurs des plantes, il n'est pas à exclure que certaines des cultures associées au jatropha puissent être des plantes-hôtes secondaires pour des ravageurs du jatropha. Dans ce cas, elles peuvent constituer un réservoir pour les ravageurs de jatropha surtout qu'il a été recommandé que cette culture soit associée aux cultures vivrières afin de contribuer à assurer la sécurité alimentaire des populations, aussi bien rurales qu'urbaines (GERES, 2010). Il importe donc de bien choisir les associations culturales dans lesquelles la culture du jatropha va être intégrée afin de ne pas exacerber le problème des ravageurs.

La présente étude vise à déterminer l'impact de l'association de J. curcas avec quelques cultures vivrières du sud-Bénin sur l'infestation et les dégâts causés à cette plante par les insectes ravageurs. Il s'agit spécifiquement d'évaluer le niveau de population des insectes ravageurs sur les plants de $J$. curcas, les dégâts causés à cette plante par les insectes ravageurs ainsi que l'effet des différentes associations culturales sur le rendement de $J$. curcas. 


\section{MATÉRIEL ET MÉTHODES Milieu d'étude}

L'étude a été conduite sur le site du projet CIRAPIP-IITA installé dans le Village de Ouokon, Arrondissement de Ouinhi, Commune de Ouinhi, Département du Zou. La pluviométrie moyenne annuelle dans la commune a varié de $1250 \mathrm{~mm}$ à $1300 \mathrm{~mm}$. La commune de Ouinhi est caractérisée par un relief constitué de bas plateaux (11 à $62 \mathrm{~m}$ d'altitude), et un climat sub-équatorial qui a contribué à la formation de sols plus ou moins fertiles et portant une végétation variée (Djènontin, 2006). Le champ abritant l'essai est situé entre les latitudes $6^{\circ} 45^{\prime}$ et $7^{\circ} 28^{\prime}$ Nord, et les longitudes $2^{\circ} 15$ et $2^{\circ} 47^{\prime}$ Est, et à une altitude moyenne de $40 \mathrm{~m}$.

\section{Dispositif expérimental}

C'est un dispositif en blocs aléatoires complets avec quatre traitements et quatre répétitions, ce qui aboutit à 16 unités parcellaires. Chaque unité parcellaire mesure $20 \mathrm{~m}$ x $20 \mathrm{~m}$, soit une superficie de $400 \mathrm{~m}^{2}$. Deux blocs adjacents sont séparés l'un de l'autre par une allée de $4 \mathrm{~m}$, et au sein d'un même bloc les unités parcellaires sont-ellesaussi séparées par une allée de $4 \mathrm{~m}$. Une bande de $2 \mathrm{~m}$, non semée, a été aménagée tout autour du champ. Les quatre traitements installés se présentent comme suit: $T_{0}=$ Jatropha seul (Témoin); $T_{1}=$ Jatropha + Manioc $; T_{2}=$ Jatropha + Maïs $; T_{3}=$ Jatropha + Niébé. Les semences du maïs, du niébé et du jatropha, sont issues des récoltes effectuées par les paysans au cours de la saison précédente. Quant au manioc, les boutures proviennent des plants conservés après la récolte des tubercules. Pour toutes ces cultures, il s'agit ici des variétés locales.

\section{Description des différents traitements ou associations culturales}

Chaque unité parcellaire comportait six lignes de $J$. curcas de $20 \mathrm{~m}$ de long. De ces six lignes de $J$. curcas, deux lignes (i.e. bande de $J$. curcas) espacées de $2 \mathrm{~m}$ sont installées à chacune des extrémités. Les deux autres lignes de J. curcas (bande de J. curcas), espacées également de $2 \mathrm{~m}$, ont été installées au milieu de la parcelle. Les deux bandes de J. curcas situées de chaque extrémité de la parcelle, sont distantes de celles du milieu de la parcelle de $7 \mathrm{~m}$. Sur ces bandes de $7 \mathrm{~m}$ sont installées huit lignes de l'une des cultures vivrières, soit niébé, maïs ou manioc selon qu'il s'agisse des traitements $T_{1}, T_{2}$ ou $T_{3}$ (GERES, 2010). Ainsi, sur chaque parcelle, on dispose d'un total de 16 lignes de $20 \mathrm{~m}$ de long de la culture vivrière concernée. Quant au traitement $T_{0}$, il est constitué uniquement des six lignes de J. curcas en culture pure (monoculture).

Sur chaque parcelle, le jatropha est planté en quinconce avec un écartement de 2,5 $\mathrm{m}$ sur les lignes et $2 \mathrm{~m}$ entre les lignes. Une bande de deux lignes de $J$. curcas constitue une rangée et deux rangées de $J$. curcas sont séparées l'une de l'autre par un intervalle de 7 $\mathrm{m}$, tel que décrit précédemment. Les semis sont directs avec deux graines introduites par poquet de $2 \mathrm{~cm}$ de profondeur dans le sol. Pour le maïs, le semis est direct avec deux graines par poquet et un écartement de $0,50 \mathrm{~m}$ $\times 0,75 \mathrm{~m}$; pour le niébé, le semis est également direct avec deux graines par poquet mais avec un écartement de 0,25 $\mathrm{m} \times 0,75 \mathrm{~m}$. Quant au manioc, les boutures ont été repiquées à écartement de $1 \mathrm{~m} \times 1 \mathrm{~m}$.

\section{Entretien des parcelles}

Après la levée des plants, il a été procédé au remplacement des manquants, et la première taille de jatropha a eu lieu au moment où le pied de jatropha était suffisamment mûr avec au moins $45 \mathrm{~cm}$ de hauteur et une tige de $2 \mathrm{~cm}$ de diamètre en moyenne. Deux ou trois autres tailles ont eu lieu au cours de l'année sur les ramifications secondaires, puis tertiaires. L'avantage de la taille est de garder un port assez bas qui facilite la récolte des fruits, facteur important à prendre en compte dans la conduite d'une plantation de $J$. curcas. La taille permet aussi à la plante d'avoir beaucoup de ramifications, et donc beaucoup de graines pour un meilleur rendement. Quant aux sarclages, ils ont été entrepris dès que le besoin s'était fait sentir 
suite aux observations faites au champ. Jatropha curcas a été planté le 25 juin 2012 et les récoltes échelonnées sur deux mois ont débuté le 25 décembre 2012, soit 6 mois après le semis, lorsque les fruits ont commencé par jaunir.

Dynamique de population des principaux insectes ravageurs de jatropha dans les différentes associations culturales

L'évolution de la densité de population des principaux ravageurs des cultures de $J$. curcas a été suivie de façon hebdomadaire au niveau de chacun des quatre traitements du champ expérimental. Dès la germination des graines, une attention particulière fut accordée aux plants par un suivi hebdomadaire régulier afin de repérer l'apparition des insectes nuisibles ou bénéfiques. Au cours de chaque séance de collecte, 30 plants de J. curcas (hormis ceux des bordures) ont été choisis au hasard par unité parcellaire (soit 120 plants par traitement), et les insectes ont été comptés visuellement sur tous les organes des plants qui les abritaient. Ceci a été fait avec beaucoup de délicatesse pour ne bousculer les plants qu'au minimum. De même, sur chacun des 30 plants échantillonnés, le pourcentage de feuilles attaquées a été évalué; le pourcentage d'attaque étant le nombre de feuilles attaquées par rapport au nombre total de feuilles que compte la plante.

\section{Evaluation des rendements de jatropha}

L'évaluation des rendements a été faite en pesant les graines de jatropha récoltées par plant à l'aide d'une balance numérique sensible de marque 'Kitchen Scale SF-400' (charge maximale $5000 \mathrm{~g}$, charge minimale 1 $\mathrm{g}$ ), le rendement étant le poids (en g) des graines récoltées par plant. Ainsi, sur chacune des six lignes de J. curcas composant un traitement, il a été choisi au hasard 2 plants par ligne, soit au total 12 plants. Les graines issues des récoltes de chaque plant ont été pesées par plant. Le poids des graines obtenues $(X i j)$ a permis de calculer le rendement moyen $X p$ par plant selon la formule suivante:
$X p=\operatorname{avec} N t=48$

$\boldsymbol{X} \boldsymbol{p}=$ Rendement moyen en graines par plant pour le jatropha du traitement ' $p$ ' $\left(p=T_{0}, T_{1}\right.$, $T_{2}$ et $T_{3}$ ) ; le traitement ' $p$ ' considéré étant répété 4 fois.

$\boldsymbol{X} \boldsymbol{i} \boldsymbol{j}=$ Poids des graines récoltées sur chaque plant, $\boldsymbol{i}=$ numéro de la parcelle pour le même traitement, $\boldsymbol{j}=$ numéro de la plante récoltée.

$\boldsymbol{N} \boldsymbol{t}=$ Nombre total des plants pour les 4 répétitions du traitement ' $p$ ' considéré.

\section{Analyses statistiques \\ Pour chaque traitement, les} pourcentages de feuilles attaquées ont été calculés. Pour chaque espèce d'insecte recensée sur les plants de jatropha dans chaque traitement (ou association culturale), la densité moyenne par plant a été déterminée. Ensuite, l'effet des différents traitements sur les densités de population des diverses espèces d'insectes ravageurs de $J$. curcas a été déterminé en utilisant l'analyse de variance (Proc GLM) dans SAS (2009). De même, l'effet des différents traitements sur les pourcentages de feuilles attaquées a été déterminé en utilisant l'analyse de variance (Proc GLM) dans SAS (2009). Quant aux rendements par plant de jatropha, ils ont été comparés entre traitements en utilisant l'analyse de variance (Proc Anova), toujours dans SAS (2009). Avant leur utilisation pour l'analyse de variance, les densités d'insecte ainsi que les rendements en graines de jatropha ont subi une transformation en $\log _{10}(\mathrm{x}+1)$ afin d'homogénéiser leurs variances. Quant aux proportions de feuilles attaquées elles ont été transformées en utilisant l'arcsinus de leur racine-carrée (arsin (sqrt(Proportion)), afin d'homogénéiser leur variance. Pour tous ces paramètres, lorsque l'analyse de variance révèle des différences significatives, les densités d'insectes par plant, les pourcentages moyens de feuilles attaquées dans les différents traitements ainsi que le rendement en graines par plant de jatropha ont été séparés entre traitements en utilisant le test de comparaison multiple de Student-NewmanKeuls (SNK). 


\section{RÉSULTATS}

Inventaire des principaux insectes ravageurs de $J$. curcas

Les insectes ravageurs de $J$. curcas rencontrés sur le champ expérimental appartiennent principalement à quatre ordres à savoir: les lépidoptères, les coléoptères, les hémiptères et les hétéroptères. Les principales espèces observées sur les plants de jatropha se présentent comme suit:

\section{Denticera divisella Duponchel} (Lepidoptera: Pyralidae): ce papillon est essentiellement nuisible au cours du stade larvaire, la chenille attaquant l'inflorescence en y tissant des toiles de soie, et fore la tige le long de laquelle elle creuse une galerie. Le bourgeon terminal du plant de jatropha est ainsi attaqué, créant une pourriture des jeunes feuilles du sommet et des inflorescences, avant de créer la pourriture des capsules.

Aulocophora africana Lucas (Coleoptera: Chrysomelidae): il a été observé sur tous les plants et en cas de forte attaque, son effectif peut atteindre 25 insectes par plant. On l'observe au niveau de tous les organes et à tous les stades phénologiques de la plante. Il attaque surtout les jeunes plantules, préférentiellement au niveau du bourgeon terminal; racle la couche chlorophyllienne des jeunes feuilles et de la partie terminale de la tige. Ainsi, s'ensuit un dessèchement rapide et une fanaison évidente de la plante surtout à cause de la forte densité par pied de ce petit coléoptère. Ces dégâts sur les feuilles se traduisent par des nécroses suivies de perforation et une pourriture sur les capsules attaquées (Fassinou, 2009).

Calidea dregei Germar (Heteroptera : Scutelleridae): l'adulte pique la capsule, suce la sève et provoque des malformations au niveau des fruits.

Lagria vilosa Fabricius (Coleoptera : Lagriidae): l'adulte, de couleur gris métallique, attaque les feuilles de J. curcas (Fassinou, 2009).

Apion sp. (Coleoptera: Apionidae) : l'adulte consomme surtout les feuilles tendres de jatropha et du niébé.
Stomphastis thraustica Meyrick (Lepidoptera: Gracillariidae) : l'adulte est un papillon de très petite taille (un microlépidoptère); la chenille est une mineuse de feuilles qui entre à l'intérieur de la feuille et se nourrit du limbe.

Clavigralla tomentosicollis Stål (Hemiptera: Coreidae) : cette punaise suceuse des gousses vertes du niébé s'attaque aussi aux fleurs de $J$. curcas qu'elle suce et provoque leur avortement.

Dynamique de population des principaux ravageurs sur les plants de $J$. curcas dans les diverses associations culturales

Les Figures 1, 2, 3 et 4 montrent l'évolution temporale des populations des principaux ravageurs sur les plants de $J$. curcas au niveau des quatre traitements à savoir, respectivement: Jatropha seul, Jatropha+Manioc, Jatropha+Maïs et Jatropha+Niébé.

Dans le traitement Jatropha seul (Figure 1), les insectes ravageurs observés sur J. curcas sont : S. straustica, Apion sp., A. africana, C. dregei et D. divisella. Pour l'espèce $S$. thraustica, la densité a varié entre $0,42 \pm 0,14$ et 2,68 $\pm 0,31$ larves par plant, et les pics de densité ont été observés successivement aux $47^{\text {ème }}, 117^{\text {ème }}, 131^{\text {ème }}$, $145^{\text {ème }}$ et $159^{\text {ème }}$ jours après semis (JAS). Pour l'espèce Apion sp., la densité a varié entre $0,00 \pm 0,00$ et $0,42 \pm 0,06$ individu par plant, et les pics de densité ont été observés aux $54^{\text {ème }}$ et $75^{\text {ème }}$ JAS. La densité de population de l'espèce $C$. dregei a varié entre $0,00 \pm 0,00$ et $0,52 \pm 0,18$ individu par plant avec des pics de densité observés aux $75^{\text {ème }}$ et $89^{\text {ème }}$ JAS. Pour l'espèce A. africana, la densité a varié entre $0,00 \pm 0,00$ et $0,74 \pm 0,09$ insecte par plant, avec des pics successifs observés aux $75^{\text {ème }}, 96^{\text {ème }}, 110^{\text {ème }}, 124^{\text {ème }}, 138^{\text {ème }}$ et $152^{\text {ème }}$ JAS. Enfin, pour l'espèce $D$. divisella, la densité a varié entre $0,00 \pm 0,00$ et 1,38 $\pm 0,16$ larve, et les pics de densité ont été observés aux $75^{\text {ème }}$ et $103^{\text {ème }}$ JAS (Figure 1 ).

Dans le traitement Jatropha+Manioc (Figure 2), les insectes ravageurs observés sur J. curcas sont: S. straustica, Apion sp., A. 
africana, C. dregei, D. divisella, L. vilosa et C. tomentosicollis. Pour l'espèce $S$. thraustica, la densité a varié entre $0,37 \pm 0,13$ et $1,73 \pm$ 0,31 larve par plant, en passant par des pics successifs aux $47^{\text {ème }}, 117^{\text {ème }}, 131^{\text {ème }}, 145^{\text {ème }}$ et $159^{\text {ème }}$ JAS. Pour l'espèce Apion sp., la densité a varié entre $0,00 \pm 0,00$ et $0,96 \pm 0,11$ individu par plant, passant par deux pics observés aux $54^{\text {ème }}$ et $75^{\text {ème }}$ JAS. La densité de population de l'espèce $C$. dregei a varié entre $0,00 \pm 0,00$ et $0,33 \pm 0,02$ individu par plant. Pour l'espèce $A$. africana, la densité a varié entre $0,00 \pm 0,00$ et 1,27 $\pm 0,14$ insecte par plant, passant par des pics dont les plus importants ont été observés aux $75^{\text {ème }}$ et $103^{\text {ème }}$ JAS. Pour l'espèce D. divisella, la densité a varié entre $0,00 \pm 0,00$ et $0,46 \pm 0,17$ larve par plant, passant par deux pics enregistrés aux $89^{\text {ème }}$ et $103^{\text {ème }}$ JAS. La densité de population de l'espèce $L$. vilosa a varié entre $0,00 \pm 0,00$ et $0,74 \pm 0,09$ individu, passant par des pics successifs observés aux $75^{\text {ème }}, 96^{\text {ème }}, 110^{\text {ème }}, 124^{\text {ème }}$ et $138^{\text {ème }} \mathrm{JAS}$. Enfin, pour l'espèce $C$. tomentosicollis, la densité a varié entre $0,00 \pm 0,00$ et $0,58 \pm 0,26$ individu par plant.

Dans le traitement Jatropha+Maïs (Figure 3), les insectes ravageurs observés sur J. curcas sont : L. vilosa, Apion sp., A. africana, $C$. dregei et $D$. divisella. Pour l'espèce $L$. vilosa, la densité a varié entre 0,00 $\pm 0,00$ et $0,74 \pm 0,09$ individu par plant, évoluant en dents de scie avec des pics observés aux $75^{\text {ème }}, 96^{\text {ème }}, 110^{\text {ème }}, 124^{\text {ème }}$, $138^{\text {ème }}$ et $152^{\text {ème }}$ JAS. Pour l'espèce Apion sp., la densité a varié entre $0,00 \pm 0,00$ et $0,96 \pm$ 0,11 individu par plant avec un pic observé au $54^{\text {ème }}$ JAS. La densité de population de l'espèce $C$. dregei a varié entre $0,00 \pm 0,00$ et $0,06 \pm 0,17$ individu par plant, avec un pic enregistré au $103^{\text {ème }}$ JAS. Pour l'espèce $A$. africana, la densité a varié entre $0,00 \pm 0,00$ et $1,38 \pm 0,15$ insecte par plant, passant par des pics observés successivement aux $75^{\text {ème }}$, $110^{\text {ème }}, 124^{\text {ème }}$ et $138^{\text {ème }}$ JAS. Enfin pour l'espèce $D$. divisella, la densité a varié entre $0,00 \pm 0,00$ et $1,27 \pm 0,17$ larve par plant, passant par des pics observés au $75^{\text {ème }}, 89^{\text {ème }}$ et $103^{\text {ème }} \mathrm{JAS}$.
Dans le traitement Jatropha+Niébé (Figure 4), les insectes ravageurs observés sur J. curcas sont: Apion sp., A. africana, C. dregei, D. divisella et $C$. tomentosicollis. Pour l'espèce Apion sp., la densité a varié entre $0,00 \pm 0,00$ et $0,96 \pm 0,11$ individu par plant, passant par deux pics observés aux $54^{\text {ème }}$ et $75^{\text {ème }}$ JAS. La densité de population de l'espèce $C$. dregei a varié entre $0,00 \pm 0,00$ et $0,46 \pm 0,17$ individu par plant, passant par des pics observés successivement aux $75^{\text {ème }}, 89^{\text {ème }}$ et $103^{\text {ème }}$ JAS. Pour l'espèce A. africana, la densité a varié entre $0,00 \pm 0,00$ et 0,74 $\pm 0,09$ insecte par plant passant par des pics observés successivement aux $75^{\text {ème }}, 103^{\text {ème }}, 124^{\text {ème }}$ et $138^{\text {ème }}$ JAS. Pour l'espèce D. divisella, la densité a varié entre $0,00 \pm 0,00$ et 1,24 $\pm 0,14$ larve par plant, passant par des pics enregistrés successivement aux $75^{\text {ème }}, 103^{\text {ème }}$, $124^{\text {ème }}$ et $138^{\text {ème }} \mathrm{JAS}$. Enfin, pour l'espèce $C$. tomentosicollis, la densité a varié entre $0,00 \pm$ 0,00 et 0,55 $\pm 0,02$ larve par plant.

Les résultats de l'analyse de variance (Proc GLM) ont révélé que les associations culturales testées ont eu un effet significatif (dl $=3 ; P<0,0001)$ sur la densité de population de la plupart des espèces d'insectes ravageurs de $J$. curcas, à l'exception de la densité d'Apion sp. sur laquelle ces associations culturales n'ont eu aucun effet significatif $(d l=3 ; P=1,000)$.

\section{Variation de la composition spécifique de l'entomofaune inféodée à $J$. curcas au sein des différentes associations culturales}

La Figure 5 présente les densités moyennes de population des insectes ravageurs du jatropha au sein de chaque association culturale (i.e. traitement). Ainsi, au niveau du traitement Jatropha seul, la densité de population la plus élevée a été observée chez $D$. divisella avec une moyenne de 0,70 $\pm 0,02$ larve par plant de jatropha, ensuite viennent respectivement dans l'ordre décroissant en terme de densité les espèces $A$. africana, Apion sp., C. dregei, S. thraustica, L. vilosa et $C$. tomentosicolis avec, respectivement : $0,44 \pm 0,02 ; 0,35 \pm 0,02$; 
$0,15 \pm 0,02 ; 0,09 \pm 0,02 ; 0,00 \pm 0,00$ et $0,00 \pm$ 0,00 individu par plant de jatropha.

Pour le traitement Jatropha+Manioc, les densités les plus fortes ont été enregistrées chez $S$. thraustica et $A$. africana avec des moyennes respectives de $1,03 \pm 0,05$ et $0,72 \pm$ 0,03 individu par plant. Ensuite viennent dans un ordre décroissant les densités des espèces L. vilosa, Apion sp., D. divisella, $C$. tomentosicolis et $C$. dregei avec, respectivement $0,44 \pm 0,02 ; 0,35 \pm 0,02 ; 0,15$ $\pm 0,02 ; 0,05 \pm 0,02 ; 0,002 \pm 0,001$ individu par plant de jatropha.

Pour le traitement Jatropha+Maïs, les densités les plus fortes ont été enregistrées chez $A$. africana avec une moyenne de 0,70 \pm 0,02 individu par plant, suivie de L. vilosa avec une moyenne de 0,44 $\pm 0,02$ individu par plant; de Apion sp. avec une moyenne de 0,34 $\pm 0,02$ individu par plant; et de $D$. divisella avec une moyenne de 0,15 $\pm 0,02$ individu par plant. Les densités de population de $C$. dregei, de $C$. tomentosicollis et de $S$. thraustica ont été les plus faibles, voire nulles.

Pour le traitement Jatropha+Niébé, les densités les plus fortes ont été enregistrées chez $D$. divisella avec une moyenne de 0,72 \pm 0,03 individu par plant, suivie de A. africana avec une moyenne de 0,44 $\pm 0,02$ individu par plant; de Apion sp. avec une moyenne de 0,34 $\pm 0,02$ individu par plant et de $C$. dregei avec une moyenne de 0,15 $\pm 0,02$ individu par plant. Les densités de population des autres insectes (i.e. S. thraustica, L. vilosa et $C$. tomentosicollis) ont été presque nulles.

L'analyse de variance (Proc GLM), effectuée au sein de chaque association culturale a révélé que les densités de population des insectes sont significativement différentes entre les espèces d'insectes ravageurs présentes $(\mathrm{dl}=7, \mathrm{~F}=382,6$ et $\mathrm{P}<$ 0,0001 pour Jatropha seul ; $\mathrm{dl}=6, \mathrm{~F}=429,3$ et $\mathrm{P}<0,0001$ pour Jatropha+Manioc ; $\mathrm{dl}=6$, $\mathrm{F}=579,2$ et $\mathrm{P}<0,0001$ pour Jatropha+Maïs ; $\mathrm{dl}=6, \mathrm{~F}=593,9$ et $\mathrm{P}<0,0001$ pour Jatropha+Niébé).

Les classements des différentes espèces suivant le test de séparation multiple de Student-Newman-Keuls (SNK) au sein de chaque association culturale sont présentés sur la Figure 5. De façon générale, les espèces dont les populations ont été les plus élevées au niveau des quatre traitements (ou associations culturales) sont : Apion sp., A. africana et D. divisella.

Effets des associations culturales sur les dégâts causés aux feuilles de $\boldsymbol{J}$. curcas

Les taux moyens de dégâts causés (i.e. nombre de feuilles attaquées par rapport au nombre total de feuilles par plant) globalement par l'ensemble des insectes sur les plants de J. curcas dans les différentes associations culturales (ou traitements) sont de $56,0 \pm 3 \% ; 52,0 \pm 3 \% ; 47,0 \pm 3 \%$ et $44,0 \pm$ $3 \%$, respectivement pour les traitements Jatropha+Maïs, Jatropha+Niébé, Jatropha+Manioc et Jatropha seul.

Les résultats de l'analyse de variance (Proc GLM) ont montré que les traitements ont eu un effet significatif sur les taux moyens de feuilles de jatropha attaquées par les insectes ravageurs $(d l=3, F=194,4$ et $P<$ 0,0001). Le test de séparation multiple de Student-Newman-Keuls (SNK) a montré que le plus faible taux de feuilles de jatropha attaquées a été enregistré dans le traitement Jatropha seul qui est considéré comme "Traitement témoin", suivi, par ordre croissant, de Jatropha+Manioc et Jatropha+Niébé tandis que les dégâts les plus élevés ont été enregistrés dans le traitement Jatropha+Maïs.

Effet des différentes associations culturales sur le rendement en graines de $\boldsymbol{J}$. curcas

Les rendements moyens en graines de jatropha par plant (i.e. poids moyen en $g$ des graines de jatropha récoltés sur chaque plant) dans les différentes associations culturales (ou traitements) sont représentés sur la Figure 6. Ils sont de 9,77 $\pm 1,57 \mathrm{~g} ; 9,40 \pm 1,71 \mathrm{~g} ; 4,63$ $\pm 1,34 \mathrm{~g}$; et $2,25 \pm 1,10 \mathrm{~g}$ par plant, respectivement pour les traitements Jatropha seul, Jatropha+Maïs, Jatropha+Niébé et Jatropha+Manioc. Les résultats de l'analyse de variance (Proc ANOVA) ont révélé que les associations culturales (i.e. traitements) ont 
eu un effet significatif sur la productivité des plants de jatropha $(d l=3 ; F=12,24 ; \quad P<$ 0,0001). Le test de séparation multiple de Student-Newman-Keuls (SNK) a montré que les rendements les plus élevés ont été enregistrés dans les traitements Jatropha seul (traitement témoin) et Jatropha+Maïs, ces deux rendements étant statistiquement similaires. En revanche, les rendements les plus faibles ont été enregistrés dans les traitements Jatropha+Niébé Jatropha+Manioc (Figure 6).

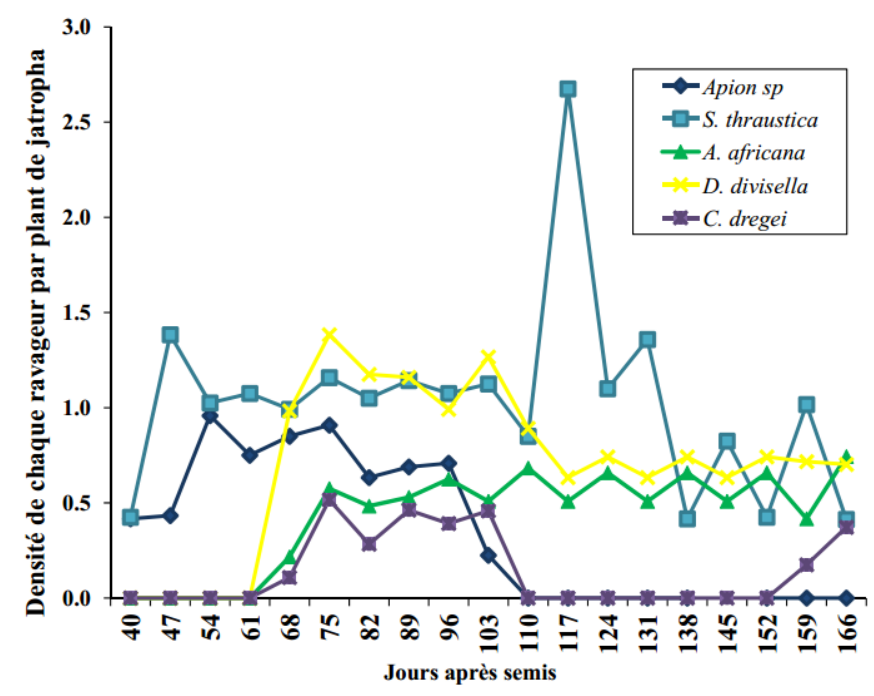

Figure 1: Fluctuations temporelles des densités par plant des principaux ravageurs de J. curcas dans le traitement Jatropha seul.

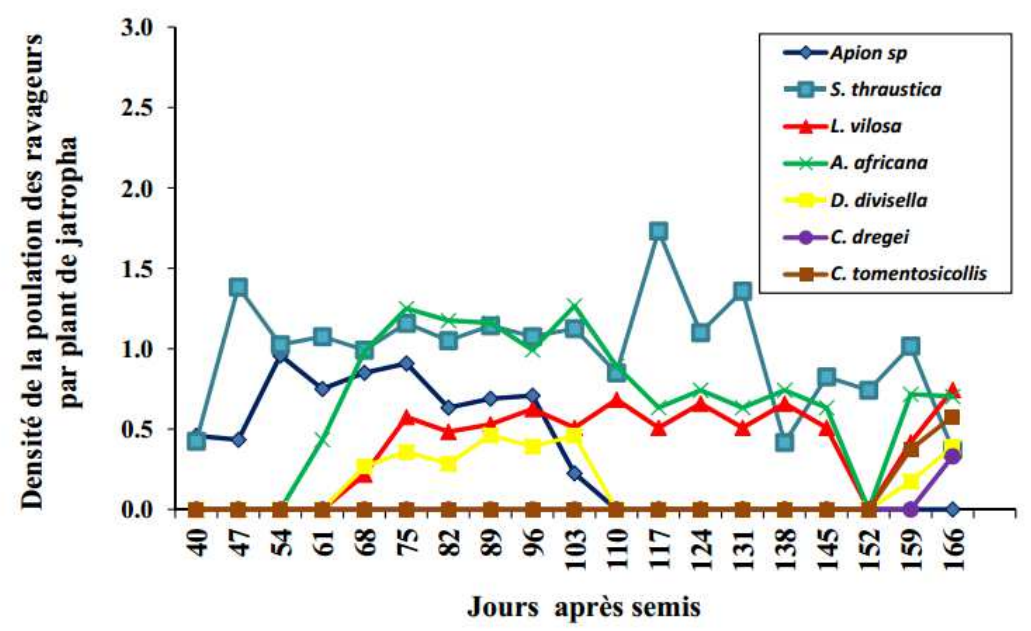

Figure 2: Fluctuations temporelles de la densité de population par plant des principaux ravageurs de J. curcas pour le traitement Jatropha+Manioc. 


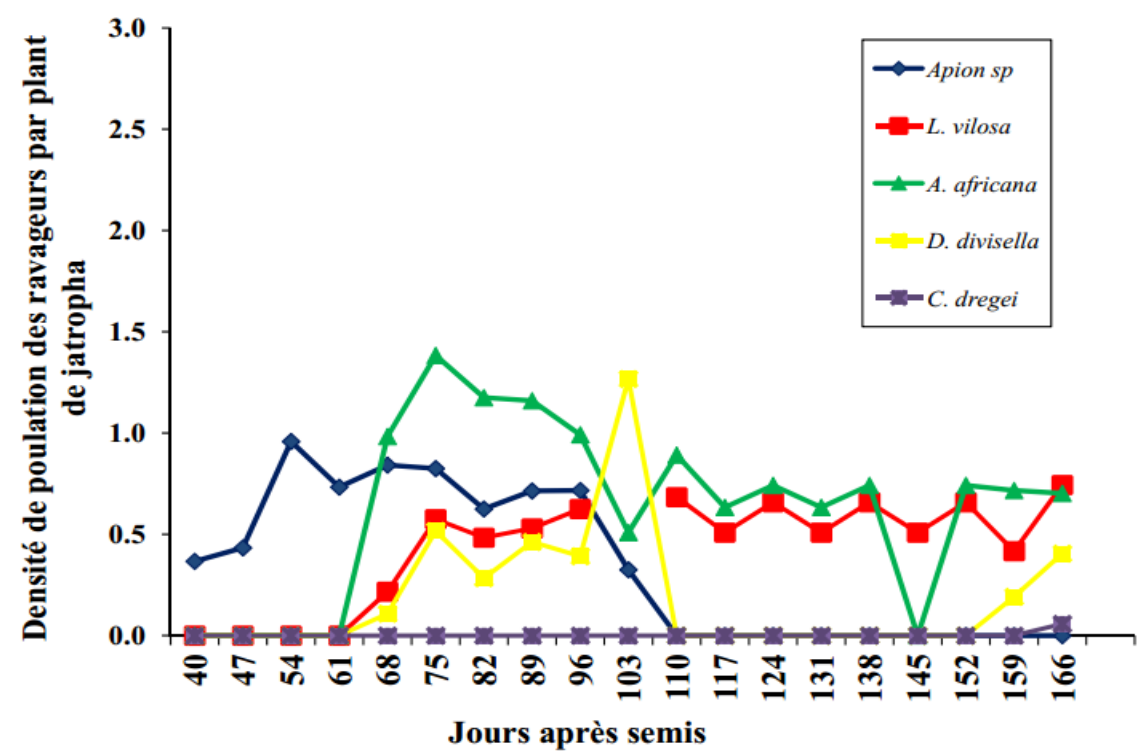

Figure 3: Fluctuations temporelles de la densité de population par plant des principaux ravageurs de J. curcas pour le traitement Jatropha+Maïs.

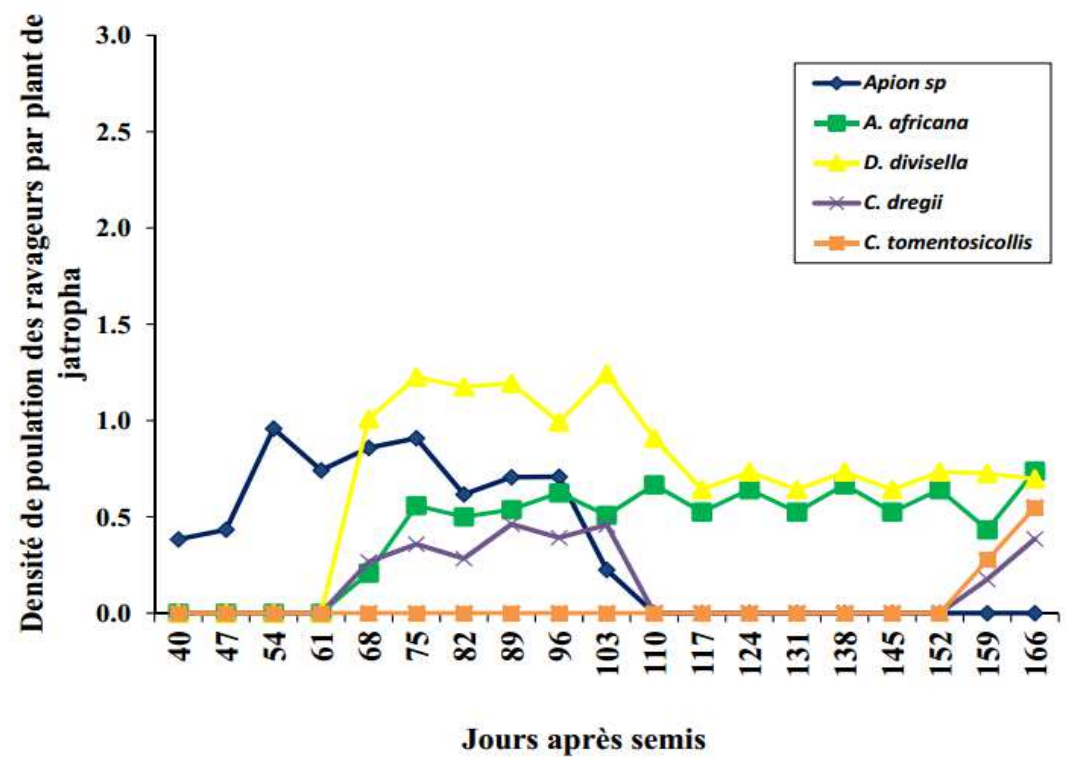

Figure 4: Fluctuations temporelles de la densité de population par plant des principaux ravageurs de J. curcas pour le traitement Jatropha+Niébé. 


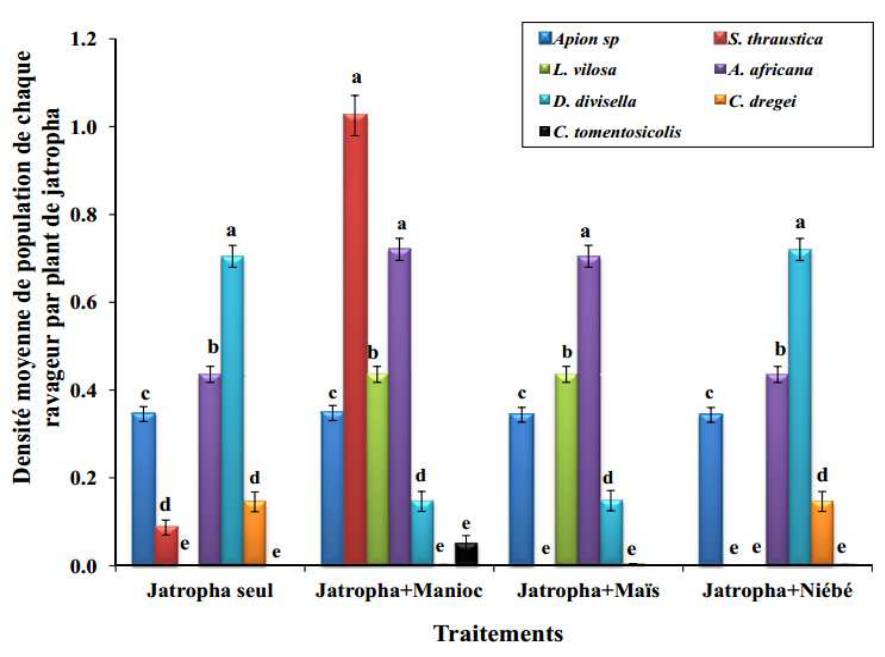

Figure 5: Densités moyennes ( \pm erreur standard) des populations des différents ravageurs de $J$. curcas par plant au sein de chaque traitement. Pour un même traitement, les barres surmontées d'une même lettre ne sont pas statistiquement différentes au seuil de 5\% selon le test de Student-Newman-Keuls (SNK).

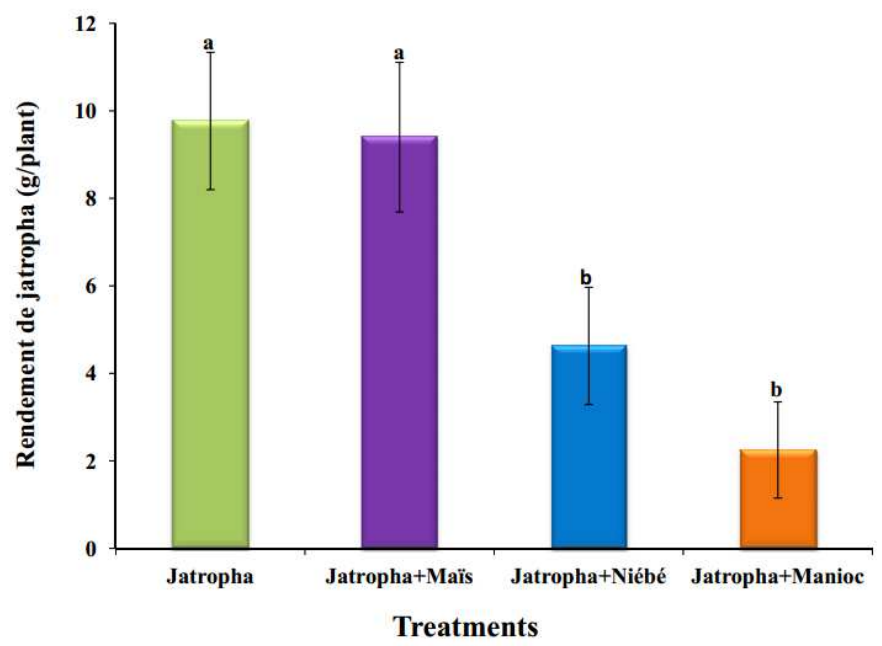

Figure 6: Rendement moyen ( \pm erreur standard) de jatropha par plant pour chaque traitement. Pour un même traitement, les barres surmontées d'une même lettre ne sont pas statistiquement différentes au seuil de 5\% selon le test de Student-Newman-Keuls (SNK).

\section{DISCUSSION}

La présente étude est à notre connaissance la première au Bénin à s'intéresser à l'aspect entomologique de l'impact de l'association culturale de $J$. curcas avec les cultures vivrières, en l'occurrence le maïs, le niébé et spécialement le manioc qui est une euphorbiacée tout comme le jatropha.
Les résultats de ce travail ont permis de recenser au niveau des quatre traitements les ravageurs suivants: $S$. straustica, Apion sp., A. africana, C. dregei, D. divisella, L. vilosa et C. tomentosicollis. Parmi ces ravageurs, seulement trois n'ont pas été cités par Togbossy (2012). Il s'agit notamment des espèces, A. africana, $L$. vilosa et $C$. tomentosicollis. Par contre, Fassinou (2009) 
avait déjà cité $A$. africana et $L$. vilosa comme étant des ravageurs de $J$. curcas. La punaise C. tomentosicollis qui n'a pas été mentionnée auparavant comme étant ravageur des fleurs $\mathrm{du}$ jatropha est traditionnellement connue comme ravageur des gousses du niébé. La présence de ce ravageur sur les plantes-hôtes autres que le niébé avait déjà été signalée par Dennis (1997) qui prévenait que ce type de ravageur peut changer de plantes hôtes durant toute l'année. Cette punaise a une préférence marquée pour les légumineuses mais a été observée attaquant Solanum incanum (Dennis, 1997). De plus, les travaux de Dabiré (2005) ont montré que tous les stades de développement de C. tomentosicollis (œufs, nymphes et adultes) ont été recensés en nombre important sur les plants de Cajanus cajan à un moment donné pendant la saison sèche et que d'autres plantes à proximité du champ de niébé telles que Acanthospermum hispidium, Borreria raddiata, Commelina forkalaei, Lucas martinicensis et Tridax procumbens ont été infestées par tous les stades de développement, mais seulement à la fin de la saison des pluies, après épuisement des gousses de niébé.

L'étude de la dynamique de population des principaux ravageurs de $J$. curcas montre que l'évolution temporelle de la densité de population des ravageurs diffère d'une espèce à une autre. Cette évolution varie aussi en fonction de l'association culturale et du stade phénologique de la plante. Ainsi, l'espèce Apion sp. qui a fait son apparition au $40^{\text {ème }}$ JAS au niveau de tous les traitements a atteint son pic au $54^{\text {ème }} \mathrm{JAS}$ pour s'annuler au $110^{\text {ème }}$ JAS. La disparition de cette espèce au $110^{\text {ème }}$ JAS pourrait s'expliquer par le fait qu'il est exclusivement un ravageur des feuilles de $J$. curcas, surtout celles qui sont encore jeunes et tendres. Quant à l'espèce A. africana, elle est apparue à partir du $61^{\text {ème }}$ JAS et sa densité de population s'est ainsi maintenue jusqu'au $166^{\text {ème }}$ JAS au niveau des quatre traitements. Cette présence de A. africana dans tous les traitements jusqu'au $166^{\text {ème }}$ JAS confirme les observations faites par Fassinou (2009) selon lesquelles ce ravageur est observé au niveau de tous les organes de la plante et est présent durant toute sa phénologie. De même, l'espèce $D$. divisella est apparue au $61^{\text {ème }}$ JAS et sa densité de population s'est maintenue jusqu'au $166^{\text {ème }}$ JAS au niveau des traitements Jatropha seul et Jatropha+Niébé, alors que dans les traitements Jatropha+Maïs et Jatropha+Manioc, ce ravageur a disparu au $110^{\text {ème }}$ JAS pour refaire surface à partir du $152^{\text {ème }}$ JAS. La présence presque constante de D. divisella sur les plants de jatropha durant toute sa phénologie se justifierait par le fait que cette espèce fait partie des ravageurs qui causent le plus de dégâts à tous les organes de $J$. curcas en l'occurrence les tiges, les fleurs, les fruits et même les feuilles. Ainsi, D. divisella se présente comme un important ravageur de jatropha. L'espèce $C$. tomentosicollis n'a été retrouvée sur les plants de $J$. curcas que dans les associations Jatropha+Niébé et Jatropha+Manioc et ce, à partir du $152^{\text {ème }} \mathrm{JAS}$, période qui correspond à la phase de floraison du jatropha et à la phase d'épuisement des gousses du niébé semé en association avec le jatropha. Ceci suppose une migration de ce ravageur du niébé vers le jatropha, confirmant ainsi les observations faites par Dennis (1997) et Dabiré (2005) comme étant l'une des conséquences de l'association culturale.

De l'analyse des données sur la composition spécifique de l'entomofaune inféodée à $J$. curcas au sein des différentes associations culturales, il ressort que les trois espèces les plus représentées en terme de densité de population au niveau des quatre traitements sont: Apion sp., A. africana et $D$. divisella. En revanche, les plus faibles densités ont été enregistrées chez les espèces C. dregei, C. tomentosicolis et L. vilosa. L'étude de l'effet des associations culturales sur les dégâts causés aux feuilles montrent que le plus faible taux de feuilles de jatropha attaquées a été enregistré dans le traitement Jatropha seul (traitement Témoin), suivi, par ordre croissant, de Jatropha+Manioc et de Jatropha+Niébé tandis que les dégâts les plus élevés ont été enregistrés dans le traitement Jatropha+Maïs. Il s'avère donc que le niveau 
des dégâts causés par les insectes sur les feuilles de J. curcas a augmenté avec l'association culturale. Ainsi, les cultures associées au jatropha constitueraient une biomasse favorable à la multiplication et au développement des insectes ravageurs qui migrent par la suite sur jatropha. Néanmoins, aucun insecte ravageur transversal n'a été enregistré entre le jatropha et le manioc qui sont pourtant tous des euphorbiacées.

De l'analyse de l'impact des associations culturales sur le rendement moyen par plant de J. curcas, il ressort que les rendements les plus élevés en graines de jatropha ont été enregistrés dans les traitements Jatropha seul $(9,77 \pm 1,57 \mathrm{~g})$ et Jatropha+Maïs $(9,40 \pm 1,71 \mathrm{~g})$, ces deux rendements étant statistiquement similaires. Par contre, les rendements les plus faibles ont été enregistrés dans les traitements Jatropha+Manioc $(4,63 \pm 1,34 \mathrm{~g})$ et Jatropha+Niébé $(2,25 \pm 1,10 \mathrm{~g})$. On en déduit que l'association culturale a eu un effet négatif sur le rendement moyen en graines de $J$. curcas, en particulier dans les associations Jatropha+Manioc et Jatropha+Niébé. Cette diminution notable de rendement serait due au niveau de population relativement élevé des ravageurs dans les associations culturales en comparaison avec Jatropha seul. Cependant, une concurrence entre $J$. curcas et les cultures associées, aussi bien dans l'occupation de l'espace que dans l'utilisation des éléments nutritifs du sol, ne saurait être exclue. Il en serait ainsi, particulièrement de l'association Jatropha+Manioc car les dégâts sur les feuilles de jatropha ont été des plus faibles sur ce traitement, ce qui n'a pas empêché que le rendement dans ce traitement soit des plus faibles. L'inverse a été néanmoins observé avec l'association Jatropha+Maïs où, malgré le niveau élevé de dégâts sur les feuilles de jatropha, les rendements en graines sont similaires à ceux obtenus avec la monoculture de jatropha. Une des explications à ce constat est que la plupart des insectes ravageurs retrouvés sur jatropha dans son association avec le maïs sont des défoliateurs (exception faite de A. africana), dont les dégâts seraient moins dommageables à jatropha que s'ils étaient majoritairement des ravageurs de fleurs, de gousses ou de graines.

Il se dégage donc de la présente étude que les associations culturales ont eu un effet négatif sur le rendement du jatropha, en particulier dans les associations Jatropha+Manioc et Jatropha+Niébé. Cette diminution de rendement ne serait certainement pas due uniquement aux dégâts commis par les insectes sur les plants de $J$. curcas mais également à l'effet négatif des cultures associées (manioc et niébé) sur la croissance et le développement du jatropha du fait de la concurrence pour certains éléments nutritifs du sol. Prudence doit donc être observée dans l'association de jatropha à d'autres cultures. Ainsi, à la lumière de la présente étude les associations Jatropha+Manioc et Jatropha+Niébé doivent être déconseillées aux producteurs.

\section{REMERCIEMENTS}

Nous témoignons aussi notre gratitude aux habitants du village de Ouokon (Commune de Ouinhi) pour leur très chaleureux accueil et leur sincère amitié. Nos remerciements vont également à l'endroit de tout le personnel de l'ONG CIRAPIP pour leur franche collaboration.

\section{RÉFÉRENCES}

Adebiyi KD. 2013. Biologie de Denticera divisella Duponchel (Lepidoptera: Pyralidae) ravageur des fleurs et fruits de Jatropha curcas L., et potentialités de son parasitoïde larvaire Syzeuctus hessei Benoît (Hymenoptera : Ichneumonidae). Mémoire d'Ingénieur Agronome, Université de Parakou, République du Bénin, 79 p.

Aponte CH. 1978. Estudio de Jatropha curcas L. como recurso biotico, University Veracruz, Xalapa-Enríquez : Veracruz, Mexico.

Brittaine R, Lutaladio N. 2010. Jatropha: a smallholder bioenergy crop. The potential for pro-poor development. Integr. Crop Manag., 8: 1- 96. 
Dabiré CLB. 2005. Effet du stade de développement des gousses de niébé sur la biologie de la punaise suceuse Clavigralla tomentosicollis (Hemiptera : coreidae). Int. J. Trop. Insect Sci., 25: 2531.

Datinon BD, Glitho AI, Tamò M, Amevoin K. 2013. Perception of farmers on seed production constraints of Jatropha curcas L. (Euphorbiaceae). Asian J. Applied Sci., 6(2): 99-106.

Dennis SH. 1997. The Economic Importance of Insects. Chapman \& Hall: London; $395 \mathrm{p}$.

Djènontin I. 2006. Monographie communale de Ouinhi. Afrique Conseil/PADC. 61 p.

Domergue M, Pirot R. 2008. Jatroph a curcas L. Rapport de synthèse bibliographique. CIRAD.

Dorin B, Gitz V. 2007. Ecobilans de biocarburants : une revue des controverses et des enjeux agronomiques mondiaux. Projet d'article formaté pour la revue Natures Sciences Sociétés. pp.337347.

Fassinou A. 2009. Gestion des ravageurs de Jatropha curcas L. par l'utilisation d'extraits botaniques naturels dans la commune de Zagnanado (Département du Zou). Thèse pour l'obtention du Master (M.Sc) en Entomologie Appliquée, Faculté des Sciences et Techniques (FAST), Université d'Abomey-Calavi, $53 \mathrm{p}$.

Gandonou C. 2007. Quelle est la situation des Agrocarburants en Afrique de l'Ouest? Semences de la biodiversité, $\mathrm{N}^{\circ} 66$ (Août 007) :1-2. (http://www.grain.org/ semences_files/note-66-fr.pdf).

GERES. 2010. Itinéraire technique expérimentale pour la production de graines de Jatropha $12 \mathrm{p}$.

Hountongninou, A. 2009. Efficacité des extraits botaniques pour la gestion des entomofaunes de Jatropha curcas L. (Euphorbiaceae) à Zagnanado (Département du Zou). Mémoire de
Licence Agricole, Université Africaine de Technologie et de Management (uatm/gasa formation), $62 \mathrm{p}$.

Kumar A, Sharma S. 2008. An evaluation of multipurpose crop for industrial uses-A review. Ind. Crop. Prod., 28 : 1-10.

Legendre B. 2008. Jatropha curcas (Tabanani). Note agronomique, $7 \mathrm{p}$.

Munch E, Kiefer J. 1986. Le Pourghère (Jatropha curcas L., Botanique, écologie, culture (1ère partie), produits de récolte, filières de valorisations, réflexions économique (2ème partie), Université de Stuttgart - Hohenheim. 276 p.

Otieno B, Mwangi L. 2005. Jatropha under attack: A host of insect pests and diseases have been reported in Kenya. Miti, nr. 1, p. 30-33.

Otim-nape GW, Bua A, Thresh, JM, Baguma Y, Ogwal S, Ssemakula GN, Acola G, Byabakama B, Colvin J, Cooter RJ, Martin A. 1997. Cassava Mosaic Virus Disease in East Africa and its Control. Natural Resources Institute: Chatham, UK; 100 p.

SAS Institute 2009. Software version 9.2 (TSMO) Cary, North Carolina, USA

Togbossy KO. 2012. Contribution à la connaissance de l'entomofaune associée à la culture du pourghère, Jatropha curcas L. (Euphorbiale : Euphorbiaceae) au Bénin. Mémoire d'Ingénieur Agronome, Ecole Supérieure d'Agronomie (ESA), Université de Lomé, 41 p.

Vignon R. 2008. Etude des techniques culturales de Jatropha curcas (Euphorbiaceae): cas des plantations expérimentales du setup dans la commune de Zagnanado. Mémoire de fin de formation pour l'obtention du diplôme de licence en agronomie. Université Africaine de Technologie et de Management (uatm/gasa formation), $40 \mathrm{p}$.

Wilbur RL. 1997. A synopsis of Jatropha, subsection Eucurcas, with the description of two new species from Mexico. $J$. Elisha Mitchell Sci. Soc., 70: 92-101. 